\title{
Ora-Pro-Nóbis: Ampliando os Conhecimentos de Sua Utilização na Alimentação a Partir da Educação Alimentar e Nutricional (EAN)
}

\author{
Ora-Pro-Nóbis: Expanding the Knowledge of Its Use in Food from Food and Nutrition \\ Education (FNE)
}

\author{
Shirlei Tessarinia; Jeferson Eduardo Pereira ${ }^{\text {b }}$, Regina Mara Silva Pereira*abc \\ aUniversidade Anhanguera de São Paulo, Programa de Pós-Graduação Stricto Sensu em Ensino de Ciência. SP, Brasil. \\ bUniversidade Anhanguera de São Paulo, Programa de Pós-Graduação Stricto Sensu em Farmácia. SP, Brasil. \\ 'Universidade Anhanguera de São Paulo, Programa de Pós-Graduação Stricto Sensu em Biotecnologia e Inovação em Saúde. SP, Brasil. \\ *E-mail: reginapereira1@anhanguera.com
}

\begin{abstract}
Resumo
Alcançar a saudabilidade por meio da mudança dos hábitos alimentares ocupa uma posição de destaque atualmente. Pesquisadores do Mundo todo buscam estudar alimentos alternativos para a manutenção da saúde e até para o tratamento de doenças. Entre os alimentos alternativos surge o Ora-Pro-Nóbis (Pereskia Aculeata Mill.), uma planta alimentícia classificada como hortaliça não convencional, que se destaca por possuir teor elevado de proteína, fibras, vitaminas e sais minerais, além de ser de baixo custo e de fácil cultivo. O presente estudo tem como objetivo apresentar os benefícios da utilização do Ora-Pro-Nóbis na dieta humana, e aprimorar a orientação sobre o seu uso junto aos profissionais da área de nutrição, contribuindo, assim, para a atuação do nutricionista como educador, por meio da prática da "Transposição Didática". Para o desenvolvimento deste artigo foi realizada uma busca bibliográfica nas bases de dados: Lilacs, SciELO, Medline, Google Acadêmico e Catálogo de Teses e Dissertações da Capes. Esse material poderá ser utilizado como base para o profissional da área de nutrição fazer uma análise comparativa da composição nutricional da hortaliça com outros alimentos convencionais consumidos pela população em geral. Dessa maneira, o educador terá informações que irão contribuir com o "saber ensinar" e a possível ampliação do "saber aprendido" dos educandos, buscando, assim, uma mudança no comportamento alimentar em prol da saúde.
\end{abstract}

Palavras-chave: Alimentos Alternativos. Plantas Alimentícias. Transposição Didática.

\begin{abstract}
Achieving healthiness through changing eating habits occupies a prominent position today. Researchers from all over the world search for alternative foods for the health maintenance and even for the treatment of diseases. Among the alternative foods, Ora-Pro-Nóbis (Pereskia Aculeata Mill.), a food plant classified as non-conventional vegetable, stands out for its high content of protein, fibers, vitamins and mineral salts, besides being of low cost and easy cultivation. This study aims to present the benefits of using Ora-Pro-Nóbis in the human diet, and improve orientation of its use with professionals in the nutrition area, thus contributing to the nutritionist's performance as an educator through the practice of "Didactic Transposition". For the development of this article, a bibliographic search was carried out in Lilacs, SciELO, Medline, Google Academic and Capes Thesis and Dissertation Catalogues. This material can be used as a basis for the nutrition professional to make a comparative analysis of the vegetables' nutritional composition with other conventional foods consumed by the general population. Thus, the educator will have information that will contribute to the "knowledge to teach" and the possible expansion of the students' "learned knowledge", thus seeking a change in food behavior in favor of health.
\end{abstract}

Keywords: Alternative Foods. Food Plants. Didatic Transposition.

\section{Introdução}

Atualmente, vive-se um período chamado de "transição nutricional", em que há o aumento na ingestão de alimentos gordurosos, refinados e ricos em açúcares e, aliado a isso, um baixo consumo de frutas, legumes e hortaliças, principalmente, as não convencionais, como a ora-pro-nóbis, que não possui uma produção agrícola em larga escala e não é amplamente conhecida pela população, mesmo que tenha uma rica composição nutricional (ROCHA et al., 2008). Em função desse tipo de alimentação se observa a diminuição das taxas de baixo peso e da desnutrição, bem como a elevação das taxas de sobrepeso e obesidade, reflexo do aumento do consumo de alimentos ultraprocessados. A inatividade física e as comodidades da vida moderna, como elevadores, escadas rolantes, controle remoto, equipamentos para transporte motorizados, e inúmeros aplicativos existentes, tornam a vida moderna incluindo a alimentação, cada vez mais rápida, dinâmica e pouco saudável.

Como consequência se verifica o aumento das taxas de doenças crônicas não transmissíveis (DCNT) diretamente ligadas aos hábitos e às práticas alimentares da população. As DCNT são importantes problemas de saúde pública. O excesso de peso, além de ser considerado uma DCNT é, também, um fator de risco para o desenvolvimento de outras doenças. Nesse período, em que são elevadas as taxas de sobrepeso e obesidade, e como resultado as DCNT, a busca pela saudabilidade se torna uma realidade. Essa busca é formada pela tríade: mudança de hábitos alimentares, qualidade de vida e saúde, obtendo melhor funcionamento global do organismo. 
Aliado a essas questões, a análise de espécies vegetais é um tema de pesquisa crescente no meio científico, com destaque para as plantas alimentícias não convencionais (PANC). Entre as PANC surge a ora-pro-nóbis, que é rica do ponto de vista nutricional, pode contribuir com a saudabilidade e a redução da fome.

Dessa forma, o presente estudo tem como objetivo apresentar a hortaliça não convencional ora-pro-nóbis e os benefícios nutricionais na dieta humana, bem como aprimorar a orientação de seu uso, contribuindo, assim, para a atuação do nutricionista como educador em saúde por meio da prática da transposição didática.

\section{Desenvolvimento}

\subsection{A prevalência das DCNT no Brasil}

Segundo dados registrados, em 2014, pela Organização Mundial da Saúde (OMS), as DCNT são responsáveis por $68 \%$ de um total de 38 milhões de mortes ocorridas no Mundo em 2012 (BRASIL, 2018). No Brasil, os dados são bastante semelhantes, conforme apontado pelo Ministério da Saúde, em 2011, as DCNT foram responsáveis por 68,3\% do total de mortes no país, havendo destaque para as doenças cardiovasculares $(30,4 \%)$, as neoplasias $(16,4 \%)$, as doenças respiratórias $(6,0 \%)$ e o diabetes $(5,3 \%)$ (BRASIL, 2018).

Existem vários fatores de risco que podem ser responsáveis pelo aparecimento das DCNT e por grande parte das mortes causadas em sua consequência, como o tabagismo, o consumo alimentar inadequado, a inatividade física e o consumo excessivo de bebidas alcoólicas (BRASIL, 2018).

\subsection{A Fome e a pobreza no Brasil}

É importante ressaltar que o oposto, ou seja, a desnutrição pela fome também causa prejuízos, que vão além das questões físicas, podendo causar danos neurológicos irreversíveis, acompanhados de distúrbios nas áreas emocionais e cognitivas, tornando o organismo ainda mais vulnerável por não conseguir desempenhar suas funções orgânicas e aptidões para um trabalho regular. Além disso, quando a situação de fome está associada à pobreza extrema e acontece nos primeiros cinco anos de vida, as consequências são graves, como o comprometimento do desenvolvimento físico, laboral e intelectual do indivíduo, o que praticamente condena esse ser humano a prejuízos irreversíveis ao desenvolvimento do sistema nervoso central (ROSANELLI et al., 2015). A mudança dos hábitos alimentares não é uma tarefa fácil, e é exatamente nesse ponto que se pode contar com a educação alimentar e nutricional.

$\mathrm{O}$ relatório "O Estado da Segurança Alimentar e Nutricional no Mundo" (Food and Nutritional Security in the World - SOFI) de 2017, (publicado em 2018), elaborado pela Food and Agriculture Organization of the United Nations (FAO), em parceria com outras organizações como: o Fundo Internacional de Desenvolvimento Agrícola (FIDA), o Fundo das Nações Unidas para a Infância (UNICEF), entre outros, mostram o aumento da fome pelo terceiro ano consecutivo.

No Caribe e na América Latina, 39,3 milhões de pessoas vivem em condições de subalimentação. Isso representa um aumento de 400 mil pessoas desde 2016. Em nível mundial, esses dados mostram que uma a cada nove pessoas foram vítimas da fome, no ano de 2017, o equivalente a 821 milhões de pessoas.

No Brasil, até 2016 a fome não havia aumentado, de forma significativa. Em 2017, tem-se a estimativa de que 2,5\% da população tenha passado fome, o que corresponde a 5,2 milhões de pessoas (GARCIA, 2018; SILVA, 2018). Além do problema da fome, é preciso avaliar e conhecer a situação de (in)segurança alimentar de uma população, o que pode dar parâmetros sobre o risco e vulnerabilidade em relação à fome. A respeito disso, um relatório, apresentado pela FAO para o PMA, informa que cerca de 113 milhões de pessoas em 53 países tiveram insegurança alimentar aguda em 2018: dados melhores que os de 2017, que indicavam 124 milhões de pessoas nessa situação.

Contudo, outras 143 milhões de pessoas de outros 42 países estão muito próximas de enfrentar a fome aguda (FOME, 2019). Sobre o Brasil se têm dados obtidos pela Rede Brasileira de Pesquisa em Soberania e Segurança Alimentar e Nutricional (PENSSAN, 2021), que mostram que $44,8 \%$ dos lares estudados de setembro a novembro de 2020, tinham garantida a segurança alimentar e nutricional de seus moradores; 55,2\% dos lares brasileiros (116,8 milhões de pessoas) passaram por algum grau de insegurança alimentar; e 9\% (19 milhões de pessoas) vivenciaram insegurança alimentar grave, ou seja, sentiram fome. Essa condição é agravada em domicílios de área rural (12\%). Este dado de 19 milhões de brasileiros em estado de fome, no período de Pandemia do novo coronavírus, representa o dobro do valor registrado em 2009, o que sugere o retorno ao nível observado na Pesquisa Nacional por Amostra de Domicílios (PNAD), em 2004, período em que o Brasil fazia parte do Mapa da Fome (FAO, 2021). A FAO avalia esse índice como o pior obtido desde então, já que até 2013 as pesquisas mostravam a regressão da fome no país.

A insegurança alimentar aguda acontece quando uma pessoa se torna incapaz de consumir alimentos adequados, colocando em risco sua vida ou seus meios de subsistência. Já a fome crônica aparece quando uma pessoa é incapaz de consumir alimentos suficientes para manter um estilo de vida normal e ativo durante um período prolongado (FOME, 2019). As causas da insegurança alimentar e da fome podem ser diversas, entre essas estão os desastres climáticos e naturais que, em 2018, levaram 29 milhões de pessoas à insegurança alimentar aguda (FOME, 2019). Frente a essas realidades, a importância da alimentação transcende o simples fato de saciar a fome física e pauta-se no binômio quantidade e qualidade de nutrientes. 


\subsection{A inclusão das PANC na alimentação}

O conceito de alimentação muda conforme o conhecimento da população sobre os alimentos saudáveis e seus benefícios. O desejo por uma vida saudável leva as pessoas a novas formas de alimentação, tendo em vista sua funcionalidade e sustentabilidade. Nesse sentido, pode-se perceber, em vários setores, mudanças profundas que têm aumentado nas últimas décadas e levam o indivíduo ao retorno à vida natural (COSTA, 2012).

As PANC são hortaliças que já tiveram um grande consumo, em algumas regiões e/ou comunidades, e em função de mudanças no comportamento alimentar da população, passaram a ter redução na expressão econômica e social, perdendo mercado para outras hortaliças. As folhas da orapro-nóbis, que é uma PANC, com elevado valor nutricional, são ricas em aminoácidos essenciais, minerais (cálcio, magnésio, manganês e zinco) e vitaminas (A, C e ácido fólico), sendo fontes suplementares de nutrientes na dieta de muitos brasileiros. Quando comparado a outros vegetais fonte de proteínas, como o feijão preto, grão de bico e as lentilhas, o ora-pro-nóbis se sobressai pelo seu alto teor proteico (SILVA et al., 2018; ZEM et al., 2017).

Portanto, o seu valor nutricional, conforme a espécie, está relacionado a teores expressivos de sais minerais, vitaminas, fibras, carboidratos e proteínas, além do reconhecido efeito funcional. $\mathrm{O}$ resgate e a valorização dessas hortaliças na alimentação representam ganhos importantes do ponto de vista cultural, econômico, social e nutricional, considerando a tradição no cultivo (por diversas comunidades) e sua contribuição em termos de nutrição (SILVA, 2017). Trata-se de uma questão de segurança e de soberania alimentar estimular a produção e o consumo das plantas não convencionais, em vista de suas características nutracêuticas e da sua rusticidade de cultivo (BRASIL, 2010).

Resultados inovadores, como a detecção de compostos bioativos responsáveis por gerar inúmeros benefícios à saúde vêm sendo apresentados sobre a qualidade biológica de espécies para fins medicinais (a cannabis) e nutracêuticas (o fitosterol) (SOUZA; SARTOR; FELIPE, 2013). As hortaliças são plantas de suma importância para o fornecimento, principalmente, de vitaminas, sais minerais e fibras, sendo que algumas dessas também são fontes de carboidratos e proteínas. Os vegetais não convencionais são alternativas alimentares em função de seu valor nutricional significativo em macro e micronutrientes, de fácil disseminação (ZEM et al., 2017).

Entre as espécies vegetais, as plantas alimentícias não convencionais (PANC) são aquelas com distribuição limitada, restrita a determinadas localidades ou regiões, exercendo grande influência na alimentação e na cultura de populações tradicionais. Além disso, são espécies que não estão organizadas, enquanto cadeia produtiva propriamente dita, diferentemente das hortaliças convencionais, como, por exemplo, a batata, o tomate, o repolho, a alface, não despertando dessa maneira, o interesse comercial por parte de empresas de sementes, fertilizantes ou agroquímicos (BRASIL, 2010).

A Pereskia aculeata Miller é uma PANC também conhecida como ora-pro-nóbis, nome proveniente do Latim (ora pro nobis), que significa "rogai por nós"; também é conhecida como "carne de homem pobre", em função de sua alta taxa de proteína (ZEM et al., 2017). É uma planta que pertence ao reino Plantae, classe Magnoliopsida, ordem Caryophyllales, família Cactaceae e gênero Pereskia (ALMEIDA; CORRÊA, 2012). É uma trepadeira de origem tropical, com seu ciclo de vida longo, podendo atingir até dez metros de altura. Essa hortaliça se apresenta na natureza com as seguintes características: suas folhas são de cor verdeescuro, carnudas e tenras, possuem um caule fino, com ramos alongados e repleta de espinhos. Podem apresentar até dez metros de altura.

No Brasil, pode ser encontrada em toda a extensão territorial entre os Estados da Bahia e do Rio Grande do Sul, principalmente, ao longo da restinga e áreas ao longo do litoral do mar pedregoso (AGOSTINI-COSTA et al., 2014). As PANC representam um papel importante também na expressão cultural de determinadas populações: o orapro-nóbis está presente na culinária tradicional de Sabará Minas Gerais, local no qual a planta faz parte dos hábitos alimentares da população e das manifestações culturais, com o festival do ora-pro-nóbis, comemorado anualmente; o inhame (cará) é outro exemplo que até meados da década de 1960 apresentava mercado expressivo e grande consumo pela população, fazendo parte do café da manhã (BRASIL, 2010a). Hoje, seu consumo foi reduzido, ao se comparar com outras hortaliças, como tomate e batata.

\subsection{Aplicando a educação alimentar e nutricional e a transposição didática na promoção das práticas alimentares saudáveis}

Nesse contexto, em que a promoção das práticas alimentares saudáveis prevalece, a Educação Alimentar e Nutricional (EAN) se destaca, portanto, o nutricionista possui um importante papel de educador. A EAN é reconhecida como estratégia que visa promoção e a proteção da saúde por meio da criação de hábitos e estilo de vida mais saudáveis (BRASIL, 2012a). A adoção de práticas e a promoção da saúde, por meio da orientação das escolhas saudáveis, podem advir de diferentes motivações: o modismo, preferência por uma vida mais saudável, necessidades de alteração da dieta por complicações de saúde, como o desenvolvimento de doenças cardiovasculares, e a adoção da mudança do estilo de vida em função de a princípios ideológicos, como é o caso dos vegetarianos e veganos.

As abordagens educativas e pedagógicas adotadas na Educação Alimentar Nutricional (EAN) devem privilegiar os processos ativos, que incorporem os conhecimentos e práticas 
populares, contextualizados às realidades dos indivíduos, suas famílias e grupos, possibilitando a integração permanente entre a teoria e a prática (BRASIL, 2012b).

A Educação Alimentar e Nutricional (EAN) é um campo de conhecimento e de prática contínua, permanente, transdisciplinar, Intersetorial e multiprofissional, que tem por objetivo a promoção da prática autônoma e voluntária de hábitos alimentares saudáveis.

De maneira geral, nas competências fundamentais para a formação do nutricionista, a EAN é uma área muito abrangente, alcançando todas as áreas de atuação dessa profissão.

$\mathrm{Na}$ prática, e de maneira bem direta, a EAN tem como objetivo atingir o educando, sendo ele de qualquer nível sociocultural e faixa etária, estando inserido em qualquer tipo de grupo populacional, a fim de promover hábitos alimentares saudáveis. Portanto, todo profissional nutricionista deve conhecer profundamente os nove princípios estruturantes para as ações da EAN, quais sejam: 1) a sustentabilidade social, ambiental e econômica; 2) a abordagem do sistema alimentar, em sua integralidade; 3) a valorização da cultura alimentar local e respeito à diversidade de opiniões e perspectivas, considerando a legitimidade dos saberes de diferentes naturezas; 4) a comida e o alimento como referências: valorização da culinária enquanto prática emancipatória; 5) a promoção do autocuidado e da autonomia; 6) a educação enquanto processo permanente e gerador de autonomia e participação ativa e informada dos sujeitos; 7) a diversidade nos cenários de prática; 8) intersetorialidade; e 9) o planejamento, avaliação e monitoramento das ações.

Nas ações de educação, o escopo do trabalho é direcionado para a atuação dos conhecimentos dos educandos para que, assim, eles possam desenvolver um senso crítico e a capacidade de intervir nas condutas alimentares errôneas de suas vidas e do seu meio, habilitando-os a criarem condições de autonomia sobre suas escolhas alimentares.

Para a realização da EAN, conforme mencionado anteriormente, que é um campo de conhecimento e de prática contínua, permanente, que envolve muitas ações, visando promoção da prática autônoma e voluntária de hábitos alimentares saudáveis, o nutricionista precisa desenvolver ações e estratégias adequadas às realidades, necessitando ter um profundo conhecimento do tema e, assim, contribuir para o resultado sinérgico entre as ações escolhidas para a difusão do saber (SANTOS, 2005). A difusão do saber realizada por meio da "Transposição Didática" implicará o fortalecimento das ações da EAN.

O termo "Transposição Didática" foi apresentado, inicialmente, em 1975, pelo sociólogo Michel Verret, interessado em discutir sobre a Educação. Em 1991, Yves Chevallard rediscutiu o tema, em seu livro, La Transposition Didactique: du savoir savant au savoir enseigné Chevallard (1991), que traz uma abordagem sobre a transformação do saber sábio (saber científico) ao saber a ser ensinado (quais metodologias empregar), e o saber ensinado (o educando assimilou o conhecimento e pode influenciar no meio em que vive).

A exemplo do saber sábio se têm os trabalhos desenvolvidos pelos pesquisadores sobre as propriedades terapêuticas e nutricionais da ora-pro-nóbis (GUIMARÃES, 2018), apresentados e validados pela comunidade científica.

Os saberes a ensinar são aqueles descritos, especificados no conjunto de textos oficiais (programas, propostas, instruções oficiais, comentários...) e definem os conteúdos, as normas, os métodos, por exemplo: A Base Nacional Comum Curricular (em discussão); os Parâmetros Curriculares Nacionais; os currículos dos cursos de nutrição; os Planos de Ensino das disciplinas do curso de nutrição; os materiais construídos pelos especialistas da área; os livros didáticos; os cursos de capacitação, pois nestes se discutem as instruções oficiais (por exemplo, a utilização de determinado material, a implementação do currículo ou o desenvolvimento de um projeto comum a um determinado grupo etc.), entre outros.

Na construção dessa sequência, o professor pode utilizar o material disponível, livros técnicos, artigos científicos, internet, entre outros, e propor diferentes metodologias utilizando ou não as tecnologias existentes.

Segundo Chevallard (1991), a descrição e especificação dos "saberes a ensinar" é feita pela noosfera, ou seja, por agentes que pensam os conteúdos de ensino, como os universitários que se interessam por assuntos educacionais, os dirigentes, os professores, as organizações como Universidades, os representantes da sociedade (por exemplo, o presidente da Associação de Nutricionistas) e os representantes políticos (o Ministro, o Secretário, seu ou de seus subordinados).

Um exemplo disso é a instituição da Política Nacional de Alimentação e Nutrição (PNAN), que pode ser considerada como uma das expressões, que oficializam a busca de uma nova direção das políticas de alimentação e nutrição ao final da década de 1990.

A PNAN, para o alcance de seus propósitos, destaca a importância em:

investir em instrumentos e estratégias de comunicação e educação em saúde que apoiem os profissionais de saúde em seu papel de socialização do conhecimento e da informação sobre alimentação e nutrição e de apoio aos indivíduos e coletividades na decisão por práticas promotoras da saúde (BRASIL, 2013, p. 23).

Essa organização que corresponde à transformação dos saberes e possibilita a inserção nas Instituições de Ensino, conduziu ao objetivo da pesquisa, que é apresentar os benefícios da utilização do ora-pro-nóbis na dieta humana, por meio da abordagem dos saberes científicos centrada na prática didática de exposição do saber sábio, construindo um material informativo sobre as propriedades nutricionais da hortaliça. Esse material poderá ser utilizado como base para o profissional fazer uma análise comparativa da composição nutricional da hortaliça com outros alimentos convencionais consumidos pela população em geral. Dessa maneira, o 
educador terá, em uma única ferramenta, informações que irão contribuir com o "saber a ensinar" e a possível ampliação do "saber aprendido" dos educandos, buscando, assim, uma mudança no comportamento alimentar em prol da saúde.

\subsection{Metodologia}

O presente artigo trata de uma revisão narrativa e para o seu desenvolvimento foi realizada uma busca bibliográfica nas bases de dados literatura latino-americana e do Caribe em Ciências da Saúde (Lilacs), Scientific Electronic Library Online (SciELO), Medical Literature Analysis and Retrieval System Online (MEDLINE), Google Acadêmico e Catálogo de Teses e Dissertações da CAPES, com os seguintes descritores: "Valor nutricional do ora-pro-nóbis"; "Ensino em saúde"; "Dieta"; e "Educação alimentar e nutricional nos anos de 2003 a 2019”. A estratégia utilizada para a busca utilizou os descritivos individualizados, e, não, em pares. A busca apresentou cerca de 78 artigos, e desses foram selecionados 20 para uma análise mais detalhada, considerando os artigos que possuíam uma relação direta com o objetivo da pesquisa.

Foram selecionados artigos científicos nos idiomas português, inglês e espanhol, e foram excluídos artigos científicos que não manifestaram interesse para o tema proposto ao desenvolvimento do artigo, como os que abordavam outras formas de plantas e hortaliças convencionais, bem como artigos publicados fora dos anos limites.

Dando continuidade, as quinze publicações selecionadas pelos descritores individualizados foram utilizadas, e os dados obtidos sobre o valor nutricional e benefícios de utilização do ora-pro-nóbis foram reunidos de maneira a se obter dados suficientes para a compilação em um único material de análise (Quadro 1).

Quadro 1 - Caracterização química de folhas e talos de ora-pro-nóbis (Pereskia aculeata Mill.) por $100 \mathrm{~g}$ de massa seca

\begin{tabular}{|c|c|c|c|c|}
\hline \multirow[b]{2}{*}{ Ensaios } & \multicolumn{2}{|c|}{$\begin{array}{c}\text { Folhas } \\
\text { Valor energético } \\
\left(173,0 \text { kcal } 100 \mathrm{~g}^{-1}\right)\end{array}$} & \multicolumn{2}{|c|}{ 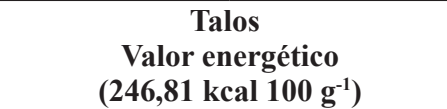 } \\
\hline & Resultado & \% V.D. (*) & Resultado & $\%$ V.D. $(*)$ \\
\hline Valor Energético & - & - & - & 12,34 \\
\hline Umidade a $105{ }^{\circ} \mathrm{C}(\%)$ & 12,89 & - & 11,95 & - \\
\hline Cinzas $(\% \mathrm{~m} / \mathrm{m})$ & 17,47 & - & 7,86 & - \\
\hline Lipídios $(\% \mathrm{~m} / \mathrm{m})$ & 4,01 & 7,29 & 4,96 & 9,0 \\
\hline Glicídios $(\% \mathrm{~m} / \mathrm{m})$ & 15,28 & 5,09 & 41,01 & 13,67 \\
\hline Proteínas $(\% \mathrm{~m} / \mathrm{m})$ & 18,95 & 25,27 & 9,53 & 12,7 \\
\hline Fibra Alimentar Total $(\% \mathrm{~m} / \mathrm{m})$ & 31,40 & 125,6 & 24,69 & 98,8 \\
\hline Sódio (mg) & 8,5 & 0,35 & 14,0 & 0,6 \\
\hline Cálcio (mg) & 105,0 & 10,5 & 107,5 & 10,7 \\
\hline
\end{tabular}

Fonte: adaptado de Rodrigues et al. (2014)

\subsubsection{Instrumento didático escolhido para transposição didática}

O instrumento escolhido para a realização da transposição didática é a elaboração de um Quadro, em que se apresenta a característica nutricional das folhas e talos da hortaliça não convencional ora-pro-nóbis.

Esse Quadro servirá como um material de estudo, fornecerá dados sobre a composição nutricional do ora-pronóbis, facilitando, assim, a formação da ação estratégica do nutricionista como educador em saúde no processo da EAN, ou seja, será possível a elaboração e cálculo nutricional da dieta ou da preparação culinária a ser apresentado ao educando.

Dessa maneira, o aconselhamento nutricional, que tem um caráter educativo, pode ser empregado pelo nutricionista com mais facilidade, já que esse terá dados coerentes e reunidos em um único material sobre a composição nutricional do orapro-nóbis. Tendo em mãos os dados apresentados no Quadro a seguir, aliado às ações estratégicas do processo de EAN, que serão adotados pelo profissional, a adesão do educando em relação ao cardápio proposto contribuirá para os objetivos relativos à mudança de comportamento alimentar.

\subsection{Propriedades nutricionais do ora-pro-nóbis}

A planta ora-pro-nóbis tem sido destacada nos experimentos científicos atuais como uma hortaliça não convencional de fácil cultivo, de baixo custo, e de elevado conteúdo nutricional. Suas folhas e seus talos são ricos em fibras, proteínas, minerais, como cálcio e vários outros componentes (Quadro 1). Esses nutrientes são de suma importância para a saúde humana, portanto, a hortaliça deve ser empregada no processo de aconselhamento nutricional individual ou em grupo (RODRIGUES et al., 2014).

O ora-pro-nóbis possui baixo valor calórico (lipídeos e glicídios), o que é esperado por se tratar de uma hortaliça. $\mathrm{O}$ baixo valor calórico é um dado importante quando se pretende abordar para o educando uma estratégia educativa focada para prevenção de DCNT, como, por exemplo, dietas para sobrepeso e obesidade.

A PANC apresenta teores elevados de umidade (água), colaborando para melhor composição de mucilagem, fator importante para ingestão como alimento e, também, para uso na indústria de produtos cosméticos (MONTEIRO, 2009). A água é parte fundamental para o bom funcionamento dos processos fisiológicos da digestão, absorção e excreção, além 
de fazer parte da composição dos tecidos corporais (MAHAN, 2005). Portanto, a água pode ser ingerida a partir dos alimentos e isso traz diversos benefícios ao organismo.

O teor de cinzas também é uma característica importante e que se destaca nas hortaliças do gênero Pereskia, pois quando os níveis são elevados, esses indicam a presença de minerais fundamentais no metabolismo do organismo. Kinupp e Barros (2008) relatam que entre as plantas alimentícias não convencionais não foram encontrados teores de cinzas maiores que os da Pereskia (17,47\% - folhas; 7,86\% - talos). Em outras hortaliças convencionais, como alface americana e brócolis, o teor de cinzas encontrados são de 9,1 \% e 7,2 \%, respectivamente. Sendo assim, o ora-pro-nóbis é reconhecido como fonte de minerais.

Além disso, a hortaliça é reconhecida por apresentar teores expressivos de proteínas, por isso chamada "carne de pobres", compostos por aminoácidos triptofano, fenilalanina, tirosina, isoleucina, leucina, treonina e lisina (ALBUQUERQUE; SABAA-SRUR; FREIMAN, 1991). Segundo a Resolução da Diretoria Colegiada (RDC) no 54, de 12 de novembro de 2012 (BRASIL, 2012a) um alimento é considerado como fonte de proteína quando apresenta teor proteico de, no mínimo, $6 \%$, e quando ultrapassa o valor de $12 \%$, poderá ser considerado de elevado valor proteico.

A proteína é um macronutriente composto por aminoácidos, que são importantes por fazerem a manutenção dos músculos, pele, tendões, glândulas, ligamentos, cabelos e até mesmo unhas. Conforme descrito no Quadro 1, a ora-pronóbis possui 18,95\% de proteína nas folhas, e 9,53\% nos talos, o que a classifica como um alimento de elevado teor proteico, ou seja, pode ser indicada para pacientes que necessitam de uma dieta restrita em proteína de origem animal.

O consumo regular das fibras está associado à prevenção de diversas desordens crônicas, que podem variar de complicações intestinais simples (obstipação) até o surgimento de úlceras, câncer do intestino grosso e do cólon, obesidade, diabetes e doenças cardíacas. Na ora-pro-nóbis são encontrados teores elevados de fibras, conforme apresentado por Silva (2018b).

Algumas hortaliças convencionais, como a alface americana e couve manteiga apresentam, respectivamente, o teor de fibras de $27,03 \%$ e $25,62 \%$, em matéria seca (TACO, 2011). O ora-pro-nóbis apresenta valores encontrados e apresentados no Quadro 1 de respectivamente 31,40\% (folhas) e 24,69\% (talos). Sabe-se que, no processo de cocção, ocorre o abrandamento das fibras, portanto o nutricionista precisa criar estratégias de EAN, cujo objetivo seja minimizar ao máximo essas perdas. A cocção a vapor e a ingestão das folhas na forma de saladas por exemplo seriam excelentes estratégias.

Outro mineral em destaque na caracterização físicoquímica do ora-pro-nóbis é o cálcio, $105 \mathrm{mg}$ nas folhas e 107,5 mg nos talos. Esses valores não estão muito distantes dos valores de referência encontrados na Tabela Brasileira de Composição de Alimentos para alimentos convencionais ricos em cálcio, como o espinafre Nova Zelândia, cru (98 mg), couve manteiga crua (131 mg) e o agrião cru (133 mg), por exemplo (TACO, 2011).

O cálcio é um nutriente fundamental para o organismo humano e seu suprimento adequado está relacionado à formação dos ossos, principalmente, nas fases da infância e adolescência. A sua presença constitui a massa óssea, prevenindo problemas de fraturas e de riscos de doenças, como a osteoporose, na vida adulta (MORAIS; BURGOS, 2007). Além das fontes como leite e seus derivados, moluscos, salmão, sardinhas e ostras, os vegetais de folhas verde-escuras também são fontes de cálcio (BUENO; CZEPIELEWSKI, 2008).

O teor de sódio da hortaliça, conforme apresentado acima, é de 8,5 mg (folhas) e $14 \mathrm{mg}$ (talos). Os valores de referência encontrados na Tabela Brasileira de Composição de Alimentos para alimentos convencionais como o espinafre Nova Zelândia, cru (120 mg), couve manteiga crua (6 mg) e o brócolis cru ( $3 \mathrm{mg}$ ). A OMS recomenda o consumo de 5 gramas de sal ao dia no máximo (TBCA, 2019).

O sódio é um nutriente importante para o organismo humano desde que seu consumo seja adequado. Esse controla contrações musculares, transmissão nervosa, manutenção da pressão arterial, equilíbrio de fluidos e ácido básico. Em excesso, está diretamente relacionado a vários prejuízos à saúde, como doenças cardiovasculares, acidente vascular cerebral, doenças renais, entre outras.

\section{Conclusão}

Por ser uma planta facilmente encontrada, de baixo custo e de rico valor nutricional, o ora-pro-nóbis é um forte aliado no combate à fome, além de colaborar no enriquecimento da dieta de vegetarianos e dietas restritas em proteína de origem animal.

Entre seus componentes nutricionais, destaca-se a proteína vegetal, as fibras e o cálcio. Esses nutrientes são poderosos para a manutenção da saúde e são fortes aliados no combate às DCNT.

A EAN, que retornou fortemente no cenário das décadas de 1990 e 2000, e proporcionou à população mais acesso à informação por meio do conhecimento das práticas alimentares, suas determinações e promoção da saúde para o combate às doenças crônicas, além do enfoque na transmissão da dimensão técnica e política, é forte aliada na disseminação de práticas alimentares saudáveis e promoção da saúde por meio da educação.

Sendo assim, faz-se necessário realizar práticas educativas junto aos profissionais e alunos de Nutrição, Gastronomia e, também, outros cursos da área Saúde, que permitam a transposição do saber sábio para o saber a ensinar na tentativa de difundir esse conhecimento, a fim de atingir o maior número de indivíduos, na forma de saber ensinado, o que pode contribuir para melhorar a qualidade de vida da população. Esses profissionais podem representar os agentes 
transformadores dos atuais hábitos alimentares da sociedade moderna.

Para tanto, é importante realizar ações como organização de palestras sobre o conhecimento científico da planta, de como utilizá-la corretamente, a fim de se manter os benefícios nutricionais durante e após o preparo, sugerir receitas e maneiras de se preparar o ora-pro-nóbis, com o intuito de promover a ampliação dos conhecimentos e uso da hortaliça, a familiarização do sabor e de seu preparo.

Ressaltamos, ainda, que as práticas utilizadas nas ações possam ser discutidas também com os educadores do Ensino Fundamental II e Ensino Médio, possibilitando-os utilizar os saberes discutidos nesse extrato de pesquisa para apresentaram projetos integrando as diferentes disciplinas e, assim, responder, de forma positiva, ao desenvolvimento de competências e habilidades indicadas na Base Nacional Comum Curricular (BNCC).

\section{Referências}

AGOSTINI-COSTA, T. S. et al. Carotenoid composition of berries and leaves from a Cactaceae - Pereskia sp. J. Functional Foods, v.11, p.178-184, 2014. doi: https://doi.org/10.1016/j. jff.2014.09.015.

AGRICULTURA familiar do Brasil é $8^{\mathrm{a}}$ maior produtora de alimentos do mundo. 12 jun. 2018. Disponível em https://www. sicoobsc.com.br/cooperando/blog/noticias/agricultura-familiarbrasil-e-8a-maior-produtora-de-alimentos-mundo/. Acesso em: 9 dez. 2020.

ALBUQUERQUE, M.G.P.T.; SABAA-SRUR, A.U.O.; FREIMAN, L.O. Composição centesimal e escore de aminoácidos em três espécies de ora-pro-nóbis (Pereskia aculeata Mill., P. bleu De Candolle e P. Pereskia (L) Karsten). Bol. SBCTA, v.25, n.1, p.7-12, 1991.

ALMEIDA, M.E.F.; CORRÊA, A.D. Utilização de cactáceas do gênero Pereskia na alimentação humana em um município de Minas Gerais. Ciênc. Rural, v.42, n.4, p.751-756, 2012. doi:10.1590/S0103-84782012000400029.

BEZERRA, T.A.; OLINDA, R.A.; PEDRAZA, D.F. Insegurança alimentar no Brasil segundo diferentes cenários sociodemográficos. Ciênc. Saúde Coletiva, v.22, n.2, p.637-651, 2017. doi: https://doi.org/10.1590/1413-81232017222.19952015.

BRASIL. Ministério da Agricultura, Pecuária e Abastecimento. Manual de hortaliças não-convencionais. Brasília: MAPA, 2010.

BRASIL. Ministério da Educação. Base Nacional Comum Curricular. Brasília: MEC, 2017.

BRASIL. Ministério da Saúde. Agência Nacional de Vigilância Sanitária. Resolução - RDC $n^{\circ} 54$, de 12 de novembro de 2012. Dispõe sobre o Regulamento Técnico sobre Informação Nutricional Complementar. Brasília: Ministério da Saúde, 2012a.

BRASIL. Ministério da Saúde. Secretaria de Atenção à Saúde. Departamento de Atenção Básica. Política nacional de alimentação e nutrição. Brasília: MS, 2012a.

BRASIL. Ministério da Saúde. Secretaria de Atenção à Saúde. Departamento de Atenção Básica. Política Nacional de Alimentação e Nutrição. Brasília: MS, 2013.

BRASIL. Ministério da Saúde. Secretaria de Vigilância em Saúde. Departamento de Análise de Situação de Saúde. Plano de ações estratégicas para o enfrentamento das doenças crônicas não transmissíveis (DCNT) no Brasil 2011-2022. Brasília: MS, 2011.

BRASIL. Ministério da Saúde. Secretaria de Vigilância em Saúde. Departamento de Vigilância de Doenças e Agravos não Transmissíveis e Promoção da Saúde. Vigitel Brasil 2017: vigilância de fatores de risco e proteção para doenças crônicas por inquérito telefônico: estimativas sobre frequência e distribuição sociodemográfica de fatores de risco e proteção para doenças crônicas nas capitais dos 26 estados brasileiros e no Distrito Federal em 2017. Brasília: MS, 2018.

BRASIL. Ministério do Desenvolvimento Social e Combate à Fome. Marco de referência de educação alimentar e nutricional para as políticas públicas. Brasília, DF: MDS, $2012 \mathrm{~b}$.

BUENO, A.L.; CZEPIELEWSKI, M.A. The importance for growth of dietary intake of calcium and vitamin D. J. Pediatrics, v.84, n.5, p.386-394, 2008. doi: https://doi.org/10.2223/ JPED. 1816 .

CHEVALLARD, Y. La transposition didactique. Grenoble: La Penséesauvage, 1991.

COSTA, E. A. Nutrição e fitoterapia: tratamento alternativo através das plantas. Petrópolis: Vozes, 2012.

DUARTE M.R.; HAYASHI, S.S. Estudo anatômico de folha e caule de Pereskia aculeata Mill. (Cactaceae). Rev Bras. Farmacog., v.15, n.2, p.103-109, 2005. doi: https://doi.org/10.1590/S0102$695 \times 2005000200006$.

FAO - Food and Agriculture Organization of the United Nations. Fome aumenta no mundo e na América Latina e no Caribe pelo terceiro ano consecutivo. 2018. Disponível em: http://www.fao. org/brasil/noticias/detail-events/en/c/1152336/. Acesso em: 6 jan. 2021.

FAO - Organização das Nações Unidas para Alimentação e Agricultura. Insegurança Alimentar e Covid-19 no Brasil. 2021b. Disponível em: http://www.fao.org/family-farming/detail/ fr/c/1392789/ . Acesso em: 5 mar. 2021.

FOME aguda afeta 113 milhões de pessoas no mundo, diz relatório da ONU. Andi, 2019. Disponível em: http://www.andi.org.br/ pauta/fome-aguda-afeta-113-milhoes-de-pessoas-no-mundodiz-relatorio-da-onu\#: : text $=\mathrm{Um} \% 20$ relat $\% \mathrm{C} 3 \% \mathrm{~B} 3$ rio $\% 20$ apresentado $\% 20$ nesta $\% 20$ ter $\% \mathrm{C} 3 \% \mathrm{~A} 7 \mathrm{a}, \mathrm{com} \% 20124 \% 20$ milh\%C3\%B5es\%20em\%202017. Acesso em: 6 jan. 2021.

GARCIA, M.F. Invisíveis e ignorados: 5,2 milhões de pessoas passam fome no Brasil. Observatório do Terceiro Setor, 2018. Disponível em: https://observatorio3setor.org.br/carrossel/ invisiveis-e-ignorados-52-milhoes-de-pessoas-passam-fome-nobrasil/. Acesso em: 6 dez. 2020.

GUIMARÃES, J.R.A. Caracterização físico-química e composição mineral de Pereskia aculeata Mill., Pereskia grandifolia Haw. e Pereskia bleo (Kunth) DC. Botucatu: UNESP, 2018.

JARDIM, L.M.; CAMARGO, S.; ZIMER, T.T.B. Transposição didática no ensino de ciências: diferentes olhares. In: CONGRESSO INTERNACIONAL DE EDUCAÇÃO, 2015, Curitiba, Paraná. Disponível em: https://educere.bruc.com.br/ arquivo/pdf2015/17323_10412.pdf. Acesso em: 6 jan. 2021.

KINUPP, V. F.; BARROS, I. B. I. Teores de proteína e minerais de espécies nativas, potenciais hortaliças e frutas. Ciênc. Tecnol. Aliment., v.28, n.4, p.846-857, 2008.

MAHAN, L. K. Minerais. In: KRAUSE, M.V.; MAHAN, L.K.; ESCOTT-STUMP, S. Alimentos, nutrição e dietoterapia. São Paulo: Roca, 2005.

MENEZES, F. A fome no Brasil é uma das facetas das desigualdades. [Entrevista cedida a] Patricia Fachin. Rev. IHU 
Online, v.31, 2017. Disponível em: http://www.ihu.unisinos. br/159-noticias/entrevistas/570090-a-fome-no-brasil-e-uma-dasfacetas-das-desigualdades-entrevista-especial-com-franciscomenezes. Acesso em: 6 dez. 2020.

MONTEIRO, B. A. Valor nutricional de partes convencionais e não convencionais de frutas e hortaliças. Botucatu: Universidade Estadual Paulista, 2009.

MORAIS, G.Q.; BURGOS, M.G.P.A. Impacto dos nutrientes na saúde óssea: novas tendências. Rev. Bras. Ortop., v.42, n.7, p.189-194, 2007. doi: https://doi.org/10.1590/S010236162007000700002 .

PINHEIRO, D.M.; PORTO, K.R.A.; MENEZES, M.E.S. A química dos alimentos: carboidratos, lipídios, proteínas e minerais. Maceió: EDUFAL, 2005.

ROCHA, D.R.C. et al. Macarrão adicionado de ora-pro-nóbis (Pereskia aculeata Miller) desidratado. Aliment. Nutr., v.19, n.4, p. 459-465, 2008.

RODRIGUES, S. et al. Caracterização química e nutricional da farinha de ora-pro-nóbis (Pereskia aculeata Mill.). Rev. Cient. Eletr. FAEF Agron., n.26, 2014. Disponível em: http://faef.revista. inf.br/site/e/agronomia-26-edicao-dezembro-de-2014.

ROSANELLI, C.F. et al. A fragilidade humana diante da pobreza e da fome. Rev. Bioética, v.23, n.1, p.89-97, 2015. doi: https://doi. org/10.1590/1983-80422015231049.
SANTOS, L.A.S. Educação alimentar e nutricional no contexto da promoção de práticas alimentares saudáveis. Rev. Nutr., v.18, n.5, 2005. doi: https://doi.org/10.1590/S1415-52732005000500011.

SILVA, D.O. et al. Acute Toxicity and Cytotoxicity of Pereskia aculeata, a Highly Nutritious Cactaceae Plant, J. Med. Food., v. 20, n.4, p.403-409, 2017. doi: 10.1089/jmf.2016.0133.

SILVA, D. O. et al. Phenological and physicochemical properties of Pereskia aculeata during cultivation in south Brazil. Horticultura Bras., v.36, n.3, p.325-329, 2018a. doi: https://doi. org/10.1590/S0102-053620180307.

SILVA, J.G.O Brasil e o mapa da fome. J. Brasil, 4 set. 2018b. Disponível em: https://www.jb.com.br/pais/artigo/2018/09/3191o-brasil-e-o-mapa-da-fome.html Acesso em: 6 dez. 2020.

SOUZA, M.C.; SARTOR, C.F.P.; FELIPE, D.F. Comparação da ação antioxidante de uma formulação contendo extrato de Pereskia aculeata com cosméticos anti-idade presentes no mercado. Rev. Saúde Pesq., v.6, n.3, p.461-477, 2013.

TBCA - Tabela Brasileira de Composição de Alimentos, versão 7.0. São Paulo: USP: FoRC, 2019.

TACO - Tabela Brasileira de Composição de Alimentos. Campinas: Unicamp, NEPA, 2011.

ZEM, L.M. et al. Pereskia aculeata: biological analysis on wistar rats. Food Sci. Technol, v.37, supl.1, p.42-47, 2017. doi: https:// doi.org/10.1590/1678-457X.29816. 